\title{
Optical coherence tomography and optical coherence tomography angiography in uveitis: A review
}

\author{
Alessandro Invernizzi MD ${ }^{1,2}$ \\ ${ }^{1}$ Department of Biomedical and Clinical Science \\ "Luigi Sacco", Eye Clinic, Luigi Sacco Hospital, \\ University of Milan, Milan, Italy \\ ${ }^{2}$ Save Sight Institute, University of Sydney, \\ Sydney, New South Wales, Australia \\ Correspondence \\ Dr Alessandro Invernizzi, Department of \\ Biomedical and Clinical Science "Luigi Sacco", \\ Eye Clinic, Luigi Sacco Hospital, University of \\ Milan, Via G. B. Grassi 74, 20157 Milan, Italy. \\ Email: alessandro.invernizzi@gmail.com
}

\author{
| Mariano Cozzi $\mathrm{MSc}^{1}$ | Giovanni Staurenghi MD ${ }^{1}$
}

Optical coherence tomography (OCT) has dramatically changed the understanding and management of uveitis and other ocular conditions. Currently, OCT angiography (OCTA) combines structural information with the visualization of blood flow within the imaged area. The aim of this review is to present the basic principles of OCT and OCTA interpretation and to investigate the role of these imaging techniques in the diagnosis and management of uveitis. Common complications of intraocular inflammation such as macular oedema and inflammatory choroidal neovascularization are often diagnosed and followed with OCT/OCTA scans. However, uveitis specialists can obtain much more information from tomographic scans. This review provides a comprehensive description of typical OCT/OCTA findings characterizing different ocular structures in uveitis, proceeding from the cornea to the choroid. A careful interpretation of OCT/OCTA images can help in the differential diagnosis, the prediction of clinical outcomes, and the follow-up of patients with uveitis.

\section{KEYWORDS}

optical coherence tomography, optical coherence tomography angiography, uveitis

\section{1 | INTRODUCTION}

Optical coherence tomography (OCT) is an imaging technique that provides in vivo quasi-histological images of the ocular tissues with a resolution beyond that of any other non-invasive technology. ${ }^{1}$ Since the introduction of OCT into clinical practice two decades ago, it has dramatically changed the understanding and the management of several ocular conditions. Currently, the structural assessment of the ocular tissues obtained through OCT images can be combined with the visualization of the blood flow within the vessels in the imaged area. The combined structural and functional images are obtained by OCT angiography (OCTA), a recent evolution of OCT technology. ${ }^{2}$

Inflammation is a response of the body to harmful stimuli. The inflammatory process promotes changes to the blood supply, the cellular components and the chemical environment of the involved area. ${ }^{3}$ These structural and functional modifications can result in transient and/or permanent changes to the tissues, both detectable with OCT $^{4}$ and OCTA. ${ }^{5}$

The aim of this review is to investigate the role of OCT and OCTA in the diagnosis and management of inflammatory ocular conditions. After a brief overview of the basic principles of these techniques, we will describe the main findings, characterizing OCT and OCTA images of the different ocular structures in uveitis, proceeding from the cornea to the choroid. Finally, we will focus on the use of OCT/OCTA in the objective quantification of intraocular inflammation and the possible future developments of these technologies.

\section{2 | OCT/OCTA BASIC PRINCIPLES}

There are several OCT technologies that vary in technical aspects, but all use a light beam to image the tissue of 
interest and to collect structural information. Spectral domain OCT (SD-OCT) is currently the most commonly used OCT technique. These instruments generate a broadband light that is split into a reference arm and a sample or "exploring" arm. As the light of the exploring arm passes through the tissues it is modified by the interaction with the different structures it encounters. Part of it is reflected back towards the source, part is absorbed, and some is scattered. The OCT device then collects the altered light from the exploring arm and compares it with the pure signal coming from the reference arm. The signal interference between the two arms is used to generate an image where the brightness varies depending on the phase relationship between the two beams. Analysis of the different frequency components of the light allows SD-OCT to simultaneously collect reflectivity information at different depths of the explored tissue (Figure 1A).

Enhanced depth imaging optical coherence tomography (EDI-OCT) is a modified acquisition procedure that allows SDOCT devices to collect images with greater details of the deeper structures, such as the choroid and the sclera. Unfortunately, this results in a concurrent decrease in the ability to visualize tissues like the vitreous and the inner retina that are closer to the light source (Figure 1B). ${ }^{6}$ Swept source optical coherence tomography (SS-OCT) is the latest generation of structural imaging. This technology is characterized by a longer wavelength and a lower axial sensitivity decay, the combination of which bypasses most of the SD-OCT and EDI-OCT limitations. Thus, SS-OCT can perform comprehensive evaluations of both the deep and the superficial structures in the same scan (Figure 1C). ${ }^{7}$

OCTA is an evolution of the OCT technology and can detect blood flow within the vessels of the explored area with no need to inject any dye. OCTA technology is based on the repetition of multiple OCT scans on the same area and the detection of differences between these scans. Because the images are acquired at very short time intervals, it is assumed that no structural changes occur between them. Consequently, any element of change between consecutive scans is interpreted by the OCTA device as "movement" and hence coded as blood flow. ${ }^{2}$

OCT images are usually evaluated on B-scans along the sagittal plane, perpendicular to the surface of the scanned structure. These cuts allow visualization of the different layers of the explored tissue along the line where the scan is performed. ${ }^{1}$ In contrast, OCTA images are usually examined following a coronal plane, parallel to the surface of the tissue of interest. This different approach is more convenient to visualize the different vascular networks that normally follow a horizontal distribution (Figure 1D,E). ${ }^{2}$

\section{3 | INTERPRETATION OF OCT IMAGES}

Uveitis includes a wide group of inflammatory responses ranging from infectious entities to autoimmune conditions
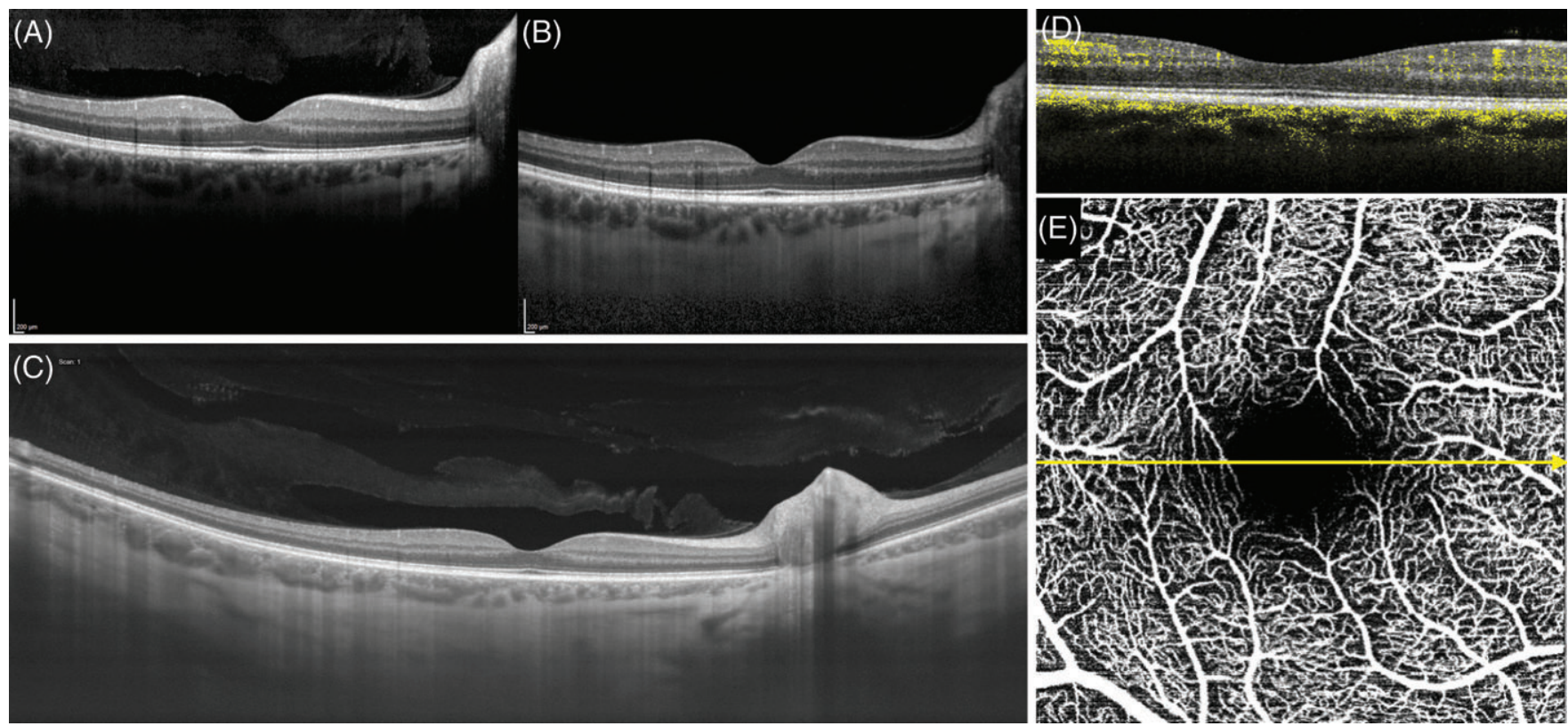

FIGURE 1 Optical coherence tomography (OCT) and OCT angiography (OCTA) scans performed at the posterior pole of the same eye with different OCT techniques. (A) OCT scan performed with spectral domain OCT (SD-OCT). The retinal layers are clearly visible as well as the details of the vitreous. Note the progressive loss of signal strength in the visualization of deeper structures (ie, choroid and sclera). (B) SD-OCT scan performed with the enhanced depth imaging modality (EDI-OCT). Differently from the previous scan, the choroid and the sclera are perfectly visualized with EDI-OCT. In contrast, vitreous details are lost. (C) Wide field scan performed with swept source OCT. This technology allows a comprehensive visualization of both the vitreous and the deeper structures. (D) OCT scan obtained with an OCTA device. The blood flow information is added to the structural information. Moving elements (flow) are usually coded in colour (yellow in this image) and correspond to the intravascular flow. (E) Coronal scan (c-scan) of the posterior pole volume obtained by OCTA. The detected signal, derived from moving elements (flow), allows visualization of the blood vessels (white). The yellow arrow represents the position of the B-scan reported in panel D 
that can target a specific ocular structure or involve the entire eye. ${ }^{3}$ The same entity can often have protean clinical presentations. In addition, different structures within the eye can be involved at the same time. This generates an overlap of OCT/OCTA findings that are often poorly described, hard to standardize, and apparently difficult to interpret. ${ }^{5}$ In such a complex scenario, the application of simple rules, based on how the OCT/OCTA images are formed, is fundamental to interpret and understand the nature of the findings.

As we previously mentioned, the OCT image is a graphic representation of the alterations that occur to the exploring light beam as it interacts with the investigated tissue. A strong reflection of the light will generate a brighter image, whereas the absorption/scattering of the OCT signal will result in a darker area on the scan. ${ }^{1}$

The way, a specific structure interacts with the OCT signal depends on several variables, the complete analysis of which goes beyond the purpose of this review. A simple rule to keep in mind is that pigments, proteins and lipids usually reflect the OCT signal, thus appearing bright on the scan (Figure 2A). By contrast, extracellular water and homogeneous structures are easily penetrated by the light and appear darker. ${ }^{8}$ Complex structures with multiple interfaces of different density such as the choroid tend to absorb and/or scatter the OCT signal. ${ }^{9}$

A final important concept is that the OCT incident light undergoes more interactions as it penetrates deeper into the tissue. For this reason, the amount of light reaching a specific layer is a direct function of the quantity of light that has been reflected/absorbed/scattered by the overlying structures. For instance, a shadowing is usually generated by hyper-reflective structures (Figure 2A), whereas the increased transmission of the signal means that the OCT light has undergone less reflection/absorption/scattering. This is usually seen when homogenous formations, such as granulomas, are imaged or when the ocular structures are thinner than usual due to atrophy (Figure 2B). ${ }^{7}$

\section{4 | INTERPRETATION OF OCTA IMAGES}

OCTA images combine the structural information of a standard OCT scan with blood flow visualization. All the reflectivity rules listed above for the structural OCT can consequently be applied to OCTA images. In addition, moving elements are coded as white pixels on the OCTA scans to represent blood flow, and this makes the interpretation more challenging. ${ }^{2}$

As mentioned above, OCTA images are usually examined following a plane parallel to the surface of the explored tissue. To create such images, the software performs an automatic segmentation of the B-scans following the reflectivity of the layers. It then separately shows the brightness information contained at specific depths. As a result, and different from traditional angiographic techniques, the images show single vascular networks contained within the explored tissue at different depths (Figure 3A,B). For this reason, while interpreting the images, it is mandatory to be aware of which
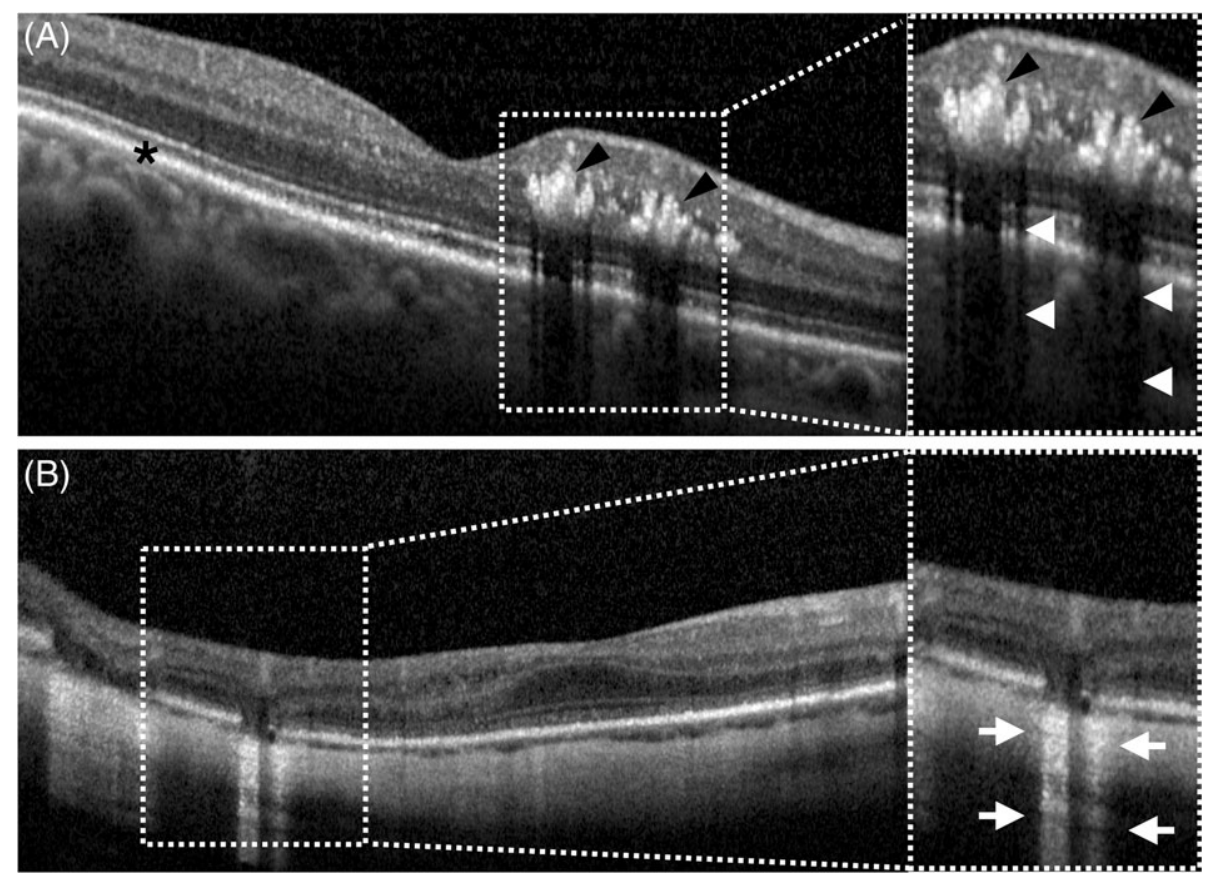

FIGURE 2 Basic interpretation of structural optical coherence tomography (OCT) image reflectivity. (A) Lipids, proteins, and pigment are highly reflective and appear bright on the OCT scan. Thus, the retinal pigment epithelium (RPE, black asterisk) and the hard exudates (black arrowheads) appear white. Highly reflective elements partially block signal penetration, inducing shadows on the underlying structures (white arrowheads). (B) In contrast, thinning of the retina or the absence of the RPE allow a higher penetration of the signal, inducing an increased transmission effect (white arrows) and better visualization of the deeper structures 
tissue layer is being shown and to double check to ascertain if the segmentation was correctly performed. ${ }^{2}$

OCTA detects the movement of erythrocytes within vessels in sequential images and then represent the changes between images as flow. However, as the moving elements affect the signal, so does the penetration of the incident light through the underlying structures, and the interaction with these structures changes over time. These changes can be wrongly interpreted by the software as movement occurring in the structures underlying the vessel. The result will be an artefact that projects the image of the vessel onto the deeper layers. $^{10}$

Finally, for technical reasons, the movement can only be detected if the velocity is within a certain range. Consequently, when the flow is either too fast or too slow, it will not be visualized, and the vascular network will appear as absent or not perfused. ${ }^{10}$

\section{I OCT/OCTA IN INTRAOCULAR INFLAMMATION: GENERAL FINDINGS}

Different types of uveitis often have specific OCT/OCTA findings according to the involved ocular tissues and the kind of inflammatory/infectious process that characterizes them. ${ }^{4,5}$ However, there are some transient or permanent changes to the physiology and anatomy of the eye that can occur independently from the underlying uveitic entity.

Increased permeability of the vessel walls is one of the key features of inflammation. This results in leakage of inflammatory proteins and migration of inflammatory cells from the intravascular space towards the inflamed area. In the eye, these changes are clinically represented by the presence of flare and inflammatory cells in the anterior chamber or in the vitreous cavity. ${ }^{3}$

In uveitis, inflammatory cells floating in the anterior chamber or in the vitreous are commonly seen as hyperreflective dots on OCT scans. ${ }^{11}$ Vitreous haze is also detectable on OCT images as an increased brightness of the vitreous cavity. ${ }^{12}$ Variations in the brightness can be used to monitor the inflammatory status of the eye (Figure 4A,B). The presence of dense vitritis or vitreous aggregates often impairs the collection of good quality OCT scans of the retina and the choroid by increasing the noise of the image and preventing the signal from reaching the posterior structures of the eye. ${ }^{4}$ Aqueous flare, in contrast, is not clearly visible on OCT images and can be evaluated only through specific algorithms of images post processing. ${ }^{11}$

Blood flow increases during inflammation. This can be detected directly by OCTA and indirectly by OCT. In the iris, OCTA can detect dilation of vessels during acute iridocyclitis, ${ }^{5}$ whereas retinal microvascular changes are visible in acute uveitis. ${ }^{13}$ An increase of the choroidal thickness can also be seen on OCT scans in most cases of uveitis, including those mainly affecting the anterior segment. ${ }^{14}$ This is an indirect measurement of increased blood flow within the choroid induced by the inflammation and can be monitored to evaluate the inflammatory status of the eye over time (Figure 4C,D).

Inflammation of the optic nerve head is common in uveitis, and the consequent swelling of the disk can be easily assessed and monitored with OCT (Figure 4E,F). ${ }^{15}$ In addition, OCTA can detect an increase in vessel density in the disc area during acute inflammation. ${ }^{16}$

One of the most common complications of uveitis is cystoid macular oedema (CME). ${ }^{17}$ This collection of fluid in the interstitial space in the macular region is hard to evaluate
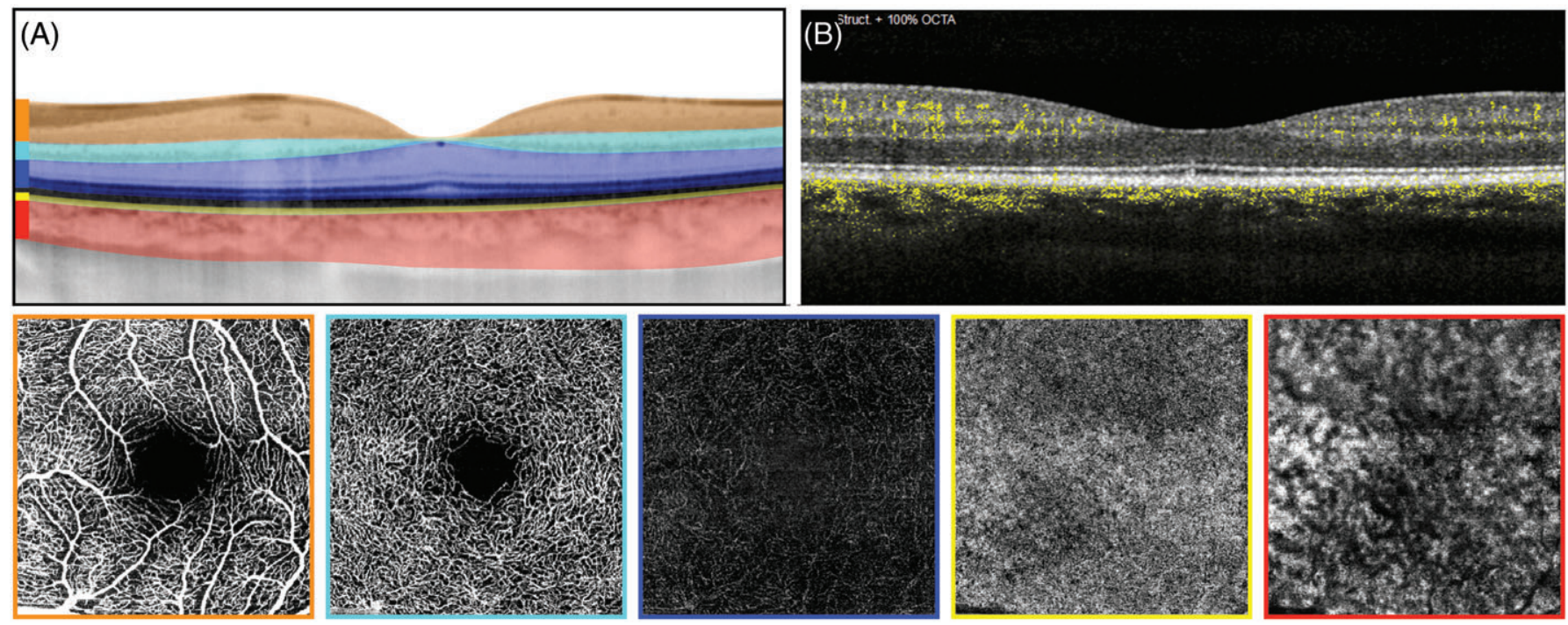

FIGURE 3 Basic interpretation of optical coherence tomography angiography (OCTA) scans. (A) Structural OCT scan of a normal eye. Coloured bands indicate the different layers analysed by OCTA automatic segmentation. The different layers are presented in the bottom row. Orange: superficial vascular plexus; turquoise: deep vascular plexus; blue: avascular zone; yellow: choriocapillaris; red: choroid. (B) OCTA B-scan showing structural reflectivity information (grey scale) and coded flow data (yellow scale) 

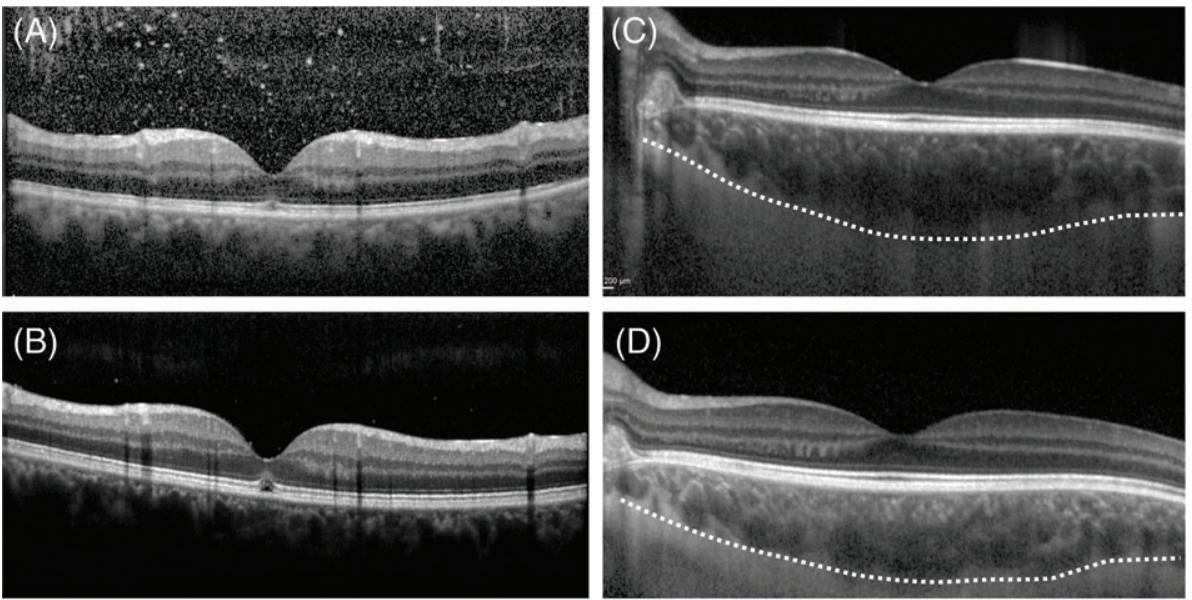
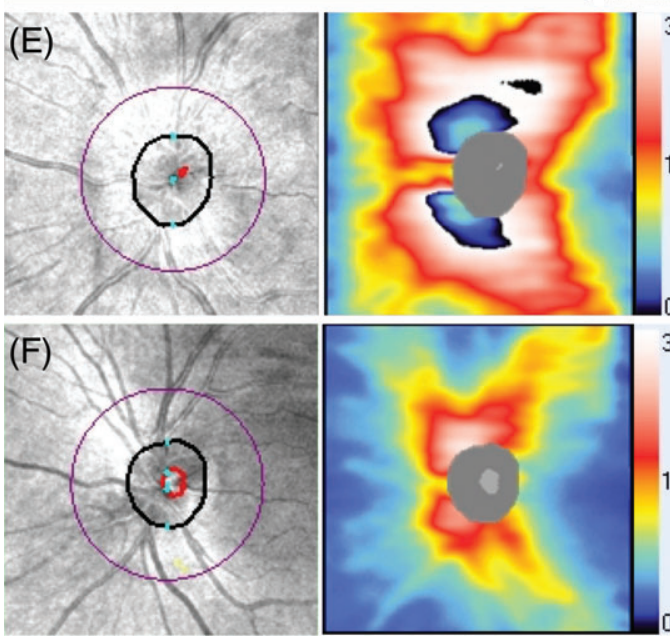
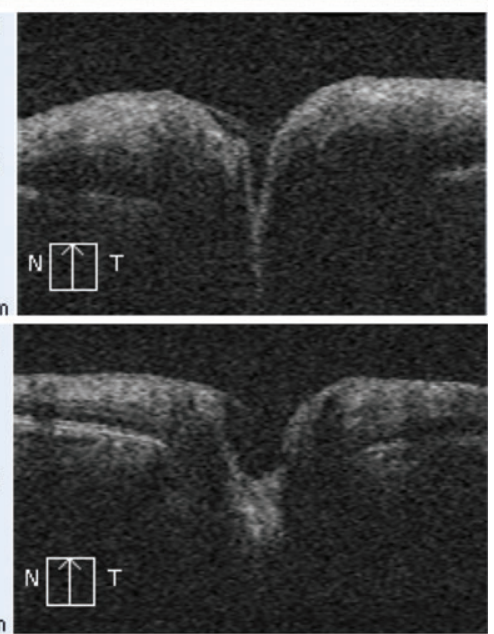

FIGURE 4 Common optical coherence tomography (OCT) findings in uveitis. (A) Vitritis is commonly seen as an increased brightness of the vitreous chamber and the presence of hyper-reflective dots in eyes with acute inflammation. (B) These findings usually disappear as the inflammation resolves. The changing in vitreous haze assessed through OCT scans can be used to monitor the disease activity. (C) Choroidal thickness is usually increased during the acute phases of uveitis and reflects increased blood flow induced by inflammation. (D) Choroidal thickness decreases and returns to normal as the inflammation disappear. The white dashed line marks the outer boundaries of the choroid in panel C and D (E) Optic nerve head inflammation is one of the most common findings in uveitis. This is visualized by OCT as an increase in thickness visible both on the colour-coded map and the B-scan. (F) The swelling can be monitored on OCT until it has resolved

through traditional biomicroscopy, especially in case of media opacity. In contrast, structural OCT easily visualizes and quantifies CME in a non-invasive way and is considered the gold standard technique for the diagnosis and follow up of this condition (Figure 5A). ${ }^{18,19}$ Often CME is accompanied by a decrease in capillary density and complexity in the macular region on OCTA. ${ }^{13}$

Chronic inflammation can induce a reaction at the level of the vitreoretinal interface, resulting in the formation of an epiretinal membrane. This complication is commonly seen in recurrent uveitis and can easily be diagnosed and followed by OCT during its evolution (Figure 5B). ${ }^{4}$

Finally, OCT and OCTA are useful tools to detect and manage inflammatory choroidal neovascularization (iCNV) (Figure 5C,D). ${ }^{4,5}$ As OCTA utilizes no dye, it does not detect leakage due to alterations of the blood retinal barrier, as traditional angiography does. ${ }^{2}$ While the absence of leakage on OCTA images may be a limitation in the detection of inflammatory changes such as exudative vasculitis, it can be an advantage in the detection of iCNVs. In fact, both iCNVs and other inflammatory lesions may show leakage on fluorescein angiography, but OCTA can easily differentiate them by visualizing the neovascular network that characterizes iCNVs. ${ }^{5}$

\section{I OCT/OCTA IN ANTERIOR UVEITIS}

Anterior uveitis (AU) is defined as an ocular inflammatory condition in which the anterior chamber of the eye is the primary site of inflammation. ${ }^{20}$ The main finding in eyes diagnosed with $\mathrm{AU}$ is the presence of cells and flare in the anterior chamber. Anterior segment OCT (AS-OCT) can be used to directly visualize and quantify the inflammatory cells floating in the aqueous humour. The cellular elements appear on the scan as hyper-reflective dots against the dark background of the anterior chamber (Figure 6A). ${ }^{21}$ In contrast, aqueous flare is usually undetectable on OCT scans, and the images need post-processing to be indirectly graded. ${ }^{11}$ 

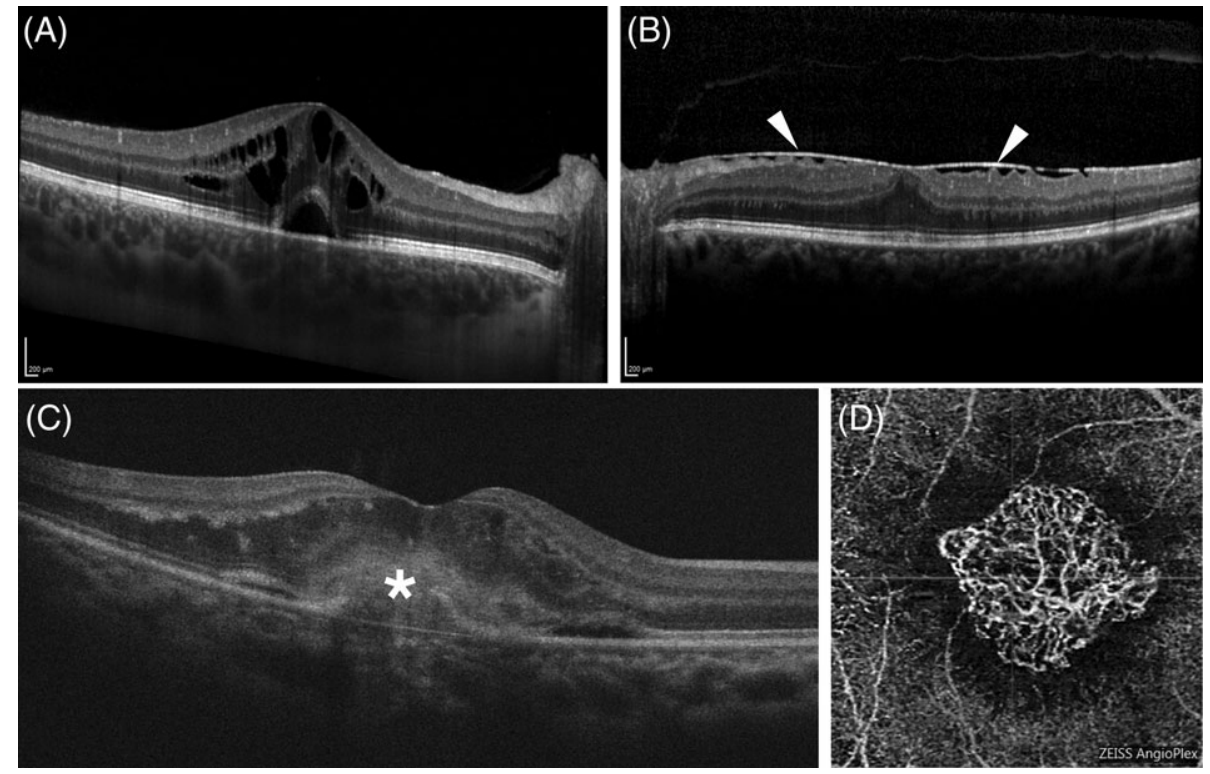

FIGURE 5 Common complications of uveitis detected and monitored with optical coherence tomography (OCT) and OCT angiography (OCTA).

(A) Cystoid macular oedema appears as a collection of fluid organized in round-shaped hypo-reflective spaces within the retina. (B) Chronic inflammation often appears as a reaction at the vitreoretinal interface and the formation of an epiretinal membrane (white arrowheads). (C) Choroidal neovascularization, a complication of uveitis, appears on structural OCT as hyper-reflective formations (white asterisk) breaking through the retinal pigment epithelium. (D) The neovascular network is readily visualized by OCTA

The deposition of inflammatory proteins on the corneal endothelium is responsible for the formation of keratic precipitates (KPs) that can have different sizes, shapes and distribution, depending on the underlying uveitic process. Recently, Kobayashi et al ${ }^{22}$ successfully used AS-OCT to characterize KPs and follow their evolution over time in cytomegalovirus (CMV) endothelitis (Figure 6B).

OCT has also been reported as a useful tool in the evaluation of iris changes induced by the inflammatory process. Mahendradas et $\mathrm{al}^{23}$ described the use of AS-OCT to monitor the response to treatment of an iris granuloma in a patient with leprosy. Independent groups reported a thinning of the iris assessed through OCT scans in patients with Fuchs uveitis. ${ }^{24,25}$ Finally, AS-OCT can be used to evaluate the presence of posterior synechia (Figure 6C) or peripheral anterior synechia in uveitic patients and help in assessing the risk to develop angle closure glaucoma. ${ }^{26}$

In $\mathrm{AU}$, the inflammatory process is mainly localized in the anterior chamber; nevertheless, recent evidence suggests that the choroidal blood flow may also be affected. Independent groups have reported that the thickness of the choroid, as measured by OCT, increases during the acute phases of AU. ${ }^{14,27}$ These changes disappeared when the inflammation was treated, suggesting that choroidal thickness assessment through OCT scans could be a useful tool in monitoring the response to therapy in these patients. ${ }^{14}$

OCTA has limited applications compared to structural OCT in AU. Dilation of the iris vessels detectable with OCTA has been reported during the acute phases of $\mathrm{AU}$, with a disappearance of the changes after treatment. ${ }^{5}$
However, the clinical relevance of these interesting findings needs further investigations.

\section{7 | OCT/OCTA IN SCLERAL/EPISCLERAL INFLAMMATION}

Inflammation of the eye walls can be investigated with OCT. Shoughy et $\mathrm{al}^{28}$ reported thickening of the sclera and the presence of hypo-reflective areas suggestive of scleral oedema on AS-OCT scans in eyes with scleritis. However, other authors reported the thickening to be visible with AS-OCT mostly in the episcleral layer in both scleritis and episcleritis. ${ }^{29}$ Similarly, another study found swelling of diffuse scleritis within the episclera rather than the scleral stroma. ${ }^{30}$ Regardless of the location of the swelling, all of the authors agree that ASOCT can be used to monitor the tissue changes over time and help in assessing the response to treatment.

There is limited literature on the use of OCTA in the assessment of the vasculature in eye walls. A recent paper from Akagi et $\mathrm{al}^{31}$ reported the possibility of using OCTA to investigate the vasculature of the normal conjunctiva and anterior sclera. Their promising results suggest possible future applications of this technology in the evaluation of patients with scleritis and episcleritis.

\section{8 | OCT/OCTA OF THE VITREOUS IN UVEITIS}

Standard SD-OCT scans of the posterior segment allow visualization of the vitreo-retinal interface, the posterior hyaloid, 

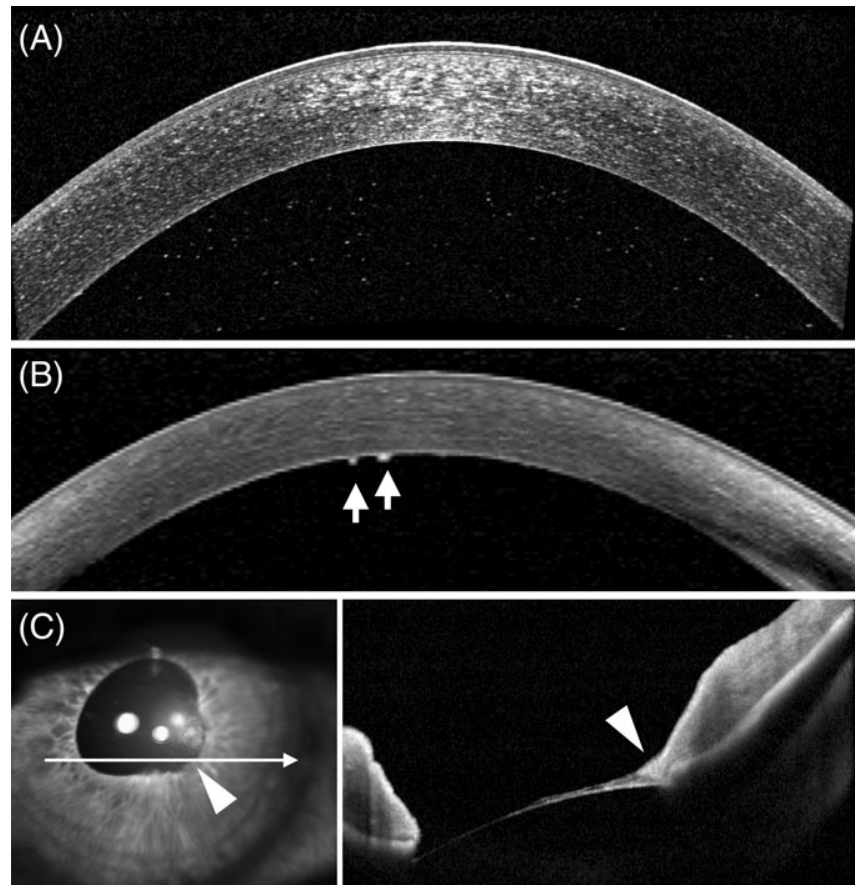

FIGURE 6 Optical coherence tomography (OCT) findings in the anterior segment of patients with uveitis. (A) On anterior segment OCT (AS-OCT) scans, inflammatory cells floating in the anterior chamber appear as hyperreflective distinct dots. (B) Keratic precipitates in a patient with Fuchs uveitis are visualized as hyper-reflective, round-shaped formations lying on the inner surface of the cornea (white arrows). (C) AS-OCT allows careful evaluation of the spatial relationships between the anterior segment structures. In this patient with sarcoidosis, the pathological adherence of the temporal pupillary margin of the iris to the lens (posterior synechia) is clearly demonstrated (white arrowhead)

and the posterior part of the vitreous body. These structures can be seen in better detail by adjusting the device's focus anterior to the retina, a technique described by Pang et al and termed "enhanced vitreous imaging." 32 SS-OCT also allows imaging of the vitreous with greater details compared to SD-OCT, making structures like the premacular bursa or the Martegnani space visible on the scans. ${ }^{33}$

The ease with which vitreous haze ${ }^{12}$ and cells ${ }^{34}$ are seen by OCT in uveitic eyes allows careful assessment of the inflammatory status of intermediate and posterior uveitis and monitoring of the response to treatment. ${ }^{35}$ A generalized increased brightness accompanied by some hyper-reflective dots are specific signs of vitreous inflammation visible on OCT of uveitic eyes.

Some diseases tend to develop unique vitritis patterns for which the OCT findings have been characterized. Invernizzi et al reported that posterior segment OCT scans of CMV retinitis patients with vitritis showed a "nebulous pattern" composed of a dusty background of increased reflectivity studded with hyper-reflective dots. In some patients this pattern was accompanied by focal areas of increased reflectivity shaped as "volcanic eruptions" rising from the points of contact between the vitreous and the necrotizing retina (Figure 7A). ${ }^{36}$
Hyper-reflective oval deposits within the vitreo-retinal interface $^{37}$ or along the detached posterior hyaloid ${ }^{38}$ have been reported during the active phase of toxoplasmic retinochoroiditis (Figure 7B). These deposits migrated within the retina and gradually disappeared as the infection was treated. $^{37}$

Finally, in patients with endogenous candida endophthalmitis, fungal aggregates lying on the retinal surface or infiltrating the vitreous appeared on OCT as round-shaped hyper-reflective lesions with a homogenous internal pattern (Figure 7C). These peculiar lesions almost completely obscured the underlying retinal layers, and thus resembled a white cloud with an underlying shower (rain-cloud sign). ${ }^{39}$

OCTA has limited applications in the vitreous imaging because there is no vascular structure at this level. However, OCTA can be useful in studying neovessels growing in response to an inflammatory stimulus in the preretinal space. Such vessels can in fact use the vitreous as a scaffold (Figure 7D,E). ${ }^{40}$ Inflammation of the vitreous is the main finding in intermediate uveitis. This is often accompanied by inflammatory changes affecting the retinal vasculature. Reduced vascular density and complexity in the retinal superficial and deep layers, along with a more heterogeneous flow signal in the choriocapillaris, have been reported on OCTA in intermediate uveitis. The authors suggested that these parameters could help in monitoring the disease and the response to treatment. ${ }^{41}$

\section{9 | OCT/OCTA OF THE RETINA IN UVEITIS}

The retina is a complex structure, with a proper vasculature divided among multiple layers. Uveitis can induce both functional and structural changes to the retina and its vessels during the acute and chronic stages of the inflammatory process. OCT and OCTA can provide useful information to detect, characterize, and monitor such changes and thereby better manage the disease.

Retinal vasculitis can be either exudative, as when the vessels show a breakdown of the blood-retinal barrier $(\mathrm{BRB})$, or occlusive, as when the inflammatory process prevents normal blood flow within the lumen. ${ }^{3}$ Exudative vasculitis is usually evaluated by fundus fluorescein angiography (FFA) because it allows visualization of dye leakage through the impaired BRB. ${ }^{42}$ Additionally, independent groups have reported that a thickening of the perivascular structures can be visualized in exudative vasculitis and monitored with structural OCT scans in patients with birdshot chorioretinopathy. ${ }^{43,44}$ Further studies are needed to establish if these findings can be observed in other uveitic entities.

Occlusive vasculitis results in retina non-perfusion, hypoxia and cells death. Structural OCT findings vary according to the stage of the inflammatory process. Acute infarcts in 

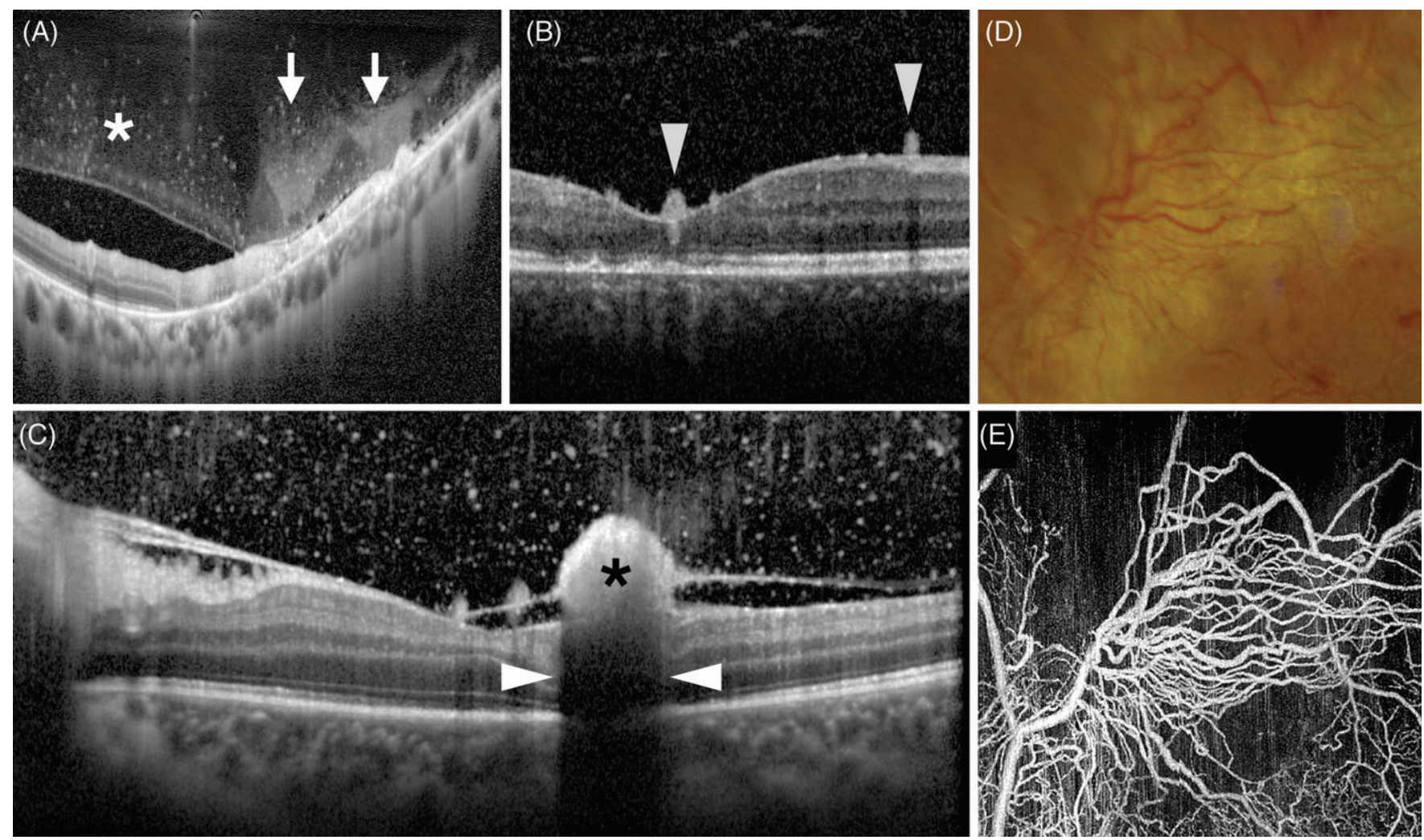

FIGURE 7 Vitreous findings on optical coherence tomography (OCT) and OCT angiography (OCTA) in patients with uveitis. (A) A specific vitritis pattern termed "nebulous vitritis" (white asterisk) and areas of increased reflectivity shaped as volcanic eruptions (white arrows) in a patient with cytomegalovirus retinitis. (B) Hyper-reflective oval deposits lying on the surface of the retina in patient with toxoplasmic chorioretinitis. (C) A fungal colony in a patient with endogenous candida endophthalmitis is visible on OCT as a round-shaped homogeneous formation (black asterisk) on the surface of the retina. The presence of the colony induces a shadowing artefact (white arrowheads) on the underlying structures (rain-cloud sign). (D, E) Epiretinal neovessels in a patient with occlusive vasculitis are visible in fundus photographs (D); however, they are better visualized with OCTA (E)

the retinal nerve fibre layer, clinically visible as cotton wool spots, appear as areas of increased thickness and reflectivity at the level of the inner retinal layers. ${ }^{45}$ A diffuse thickening, disruption of the retinal architecture, and increased reflectivity is seen in cases of retinal ischaemia. ${ }^{46}$ These changes turn into thinning or disappearance of the affected retinal layers during the chronic stage of the disease. ${ }^{45,46}$ Because OCTA is more sensitive than FFA in the imaging of ischaemic changes, it allows careful visualization and quantitation of retinal hypo-perfusion in occlusive vasculitis (Figure $8 \mathrm{~A}$ ). ${ }^{5}$ Decreased capillary density and complexity in both the superficial and deep vascular plexuses have been reported in the parafoveal area in different kinds of uveitis. ${ }^{13,41}$

Both infectious and non-infectious uveitis can be characterized by retinitis and consequent retinal necrosis. Structural OCT enables determination of which retinal layers are involved and helps in the differential diagnosis. Full thickness increases in reflectivity and retinal thickening, along with disruption of all of the retinal layers, are commonly seen in acute toxoplasmic retinochoroiditis (Figure 8B), viral retinitis (Figure $8 \mathrm{C}$ ) and Behçet posterior uveitis. ${ }^{47,48} \mathrm{Spe}$ cific patterns of findings can help to differentiate among the different entities. ${ }^{38}$ Patients with the same disease can also show different OCT alterations within the retinal layers, and these can help in predicting the outcomes and managing the disease. For instance, the identification of large empty spaces in the outer nuclear layer in eyes with CMV retinitis has been correlated with subsequent retinal detachment. ${ }^{36}$

OCTA findings in areas of retinitis have been reported in both infectious and non-infectious uveitis. The images usually allow visualization of disruption or absence of the normal capillary plexuses in the affected areas (Figure 8D). ${ }^{49,50}$ OCTA was used to detect a neovascular network within the infected focus in a case of bartonella ${ }^{51}$ and the presence of a chorioretinal anastomosis following the regression of a retinal lesion in a patient with endogenous candida endophthalmitis. ${ }^{40}$

\section{0 | OCT/OCTA OF THE OUTER RETINA, RETINAL PIGMENT EPITHELIUM AND CHORIOCAPILLARIS IN UVEITIS}

The integrity of the outer retina evaluated with OCT has been positively associated with visual function in several ocular conditions, including uveitis. Photoreceptors can be directly affected by the inflammatory process or suffer from a metabolic insult derived from a dysfunctional retinal pigment epithelium (RPE) or choriocapillaris. The involvement of the outer retina in the inflammatory process is usually 

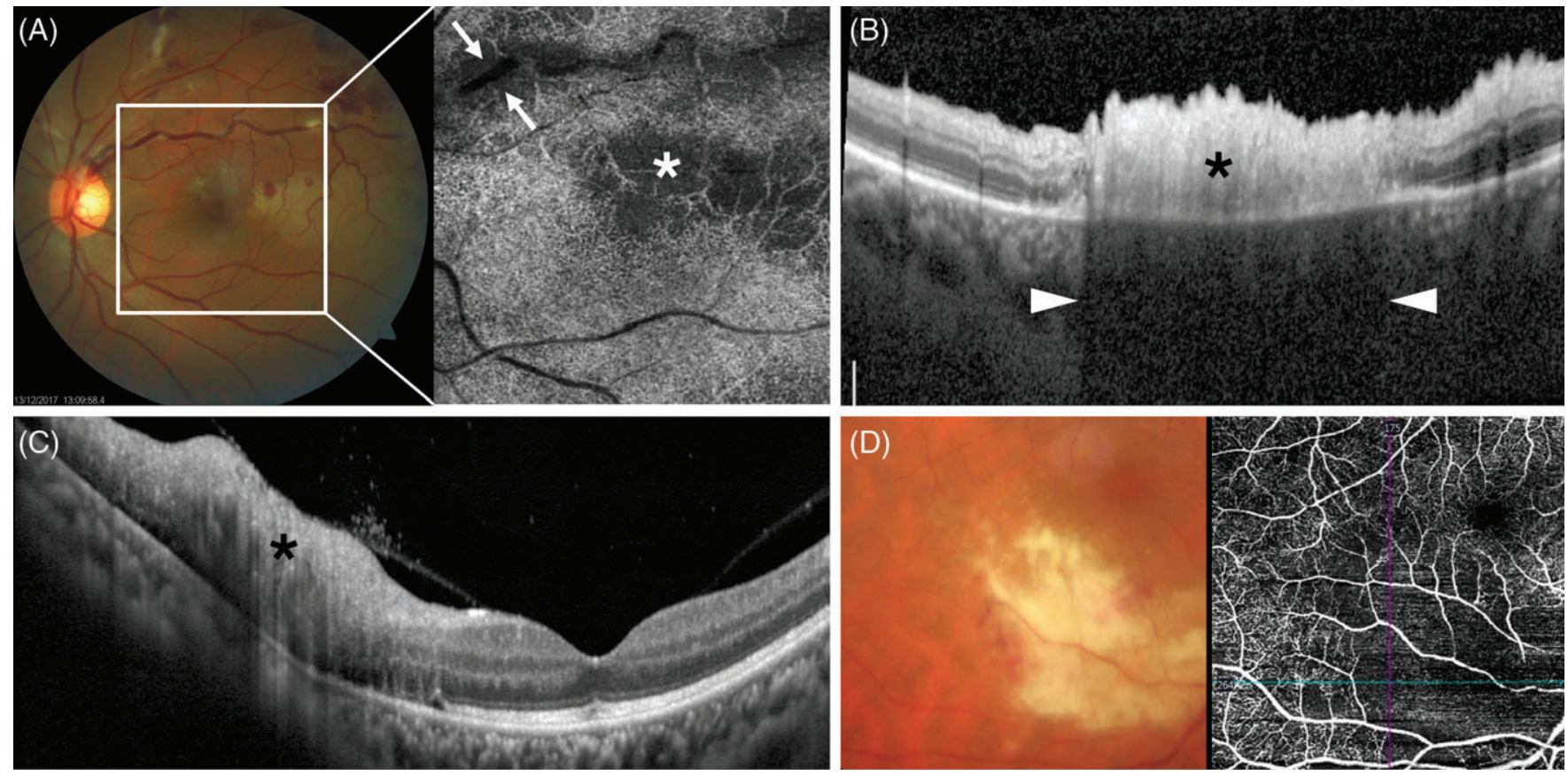

FIGURE 8 Retinal findings on optical coherence tomography (OCT) and OCT angiography (OCTA) in patients with uveitis. (A) Combined fundus photography and OCTA in a patient with tubercular-related uveitis. The temporal side of the posterior pole appears whitened, likely due to an ischaemic event secondary to occlusive vasculitis. The OCTA scan over the area demonstrates the absence of flow in the affected area (white asterisk), confirming hypoperfusion. A hypo-reflective halo is also seen along the borders of the inflamed large vein crossing the superior side of the image (white arrows). This decreased brightness is generated by the thickening of the oedematous retina beside the vessel. (B) Structural OCT scan encompassing an area of active chorioretinitis in a patient affected by toxoplasmosis. The retina appears thickened with disruption of the retinal layers (black asterisk). The area of retinitis impairs the signal penetration, inducing a shadowing on the underlying structures (white arrowheads). (C) Structural OCT scan encompassing an area of active retinitis in a patient with cytomegalovirus (CMV) uveitis. The retinal layers appear disrupted (black asterisk). Contrary to what is commonly seen in toxoplasmosis, there is only minimal shadowing, and the choroidal structure is still visible. (D) Combined fundus photography and OCTA in a patient with CMV retinitis. The OCTA shows the absence of flow in the area of active necrotizing retinitis

seen on OCT as increased reflectivity and a disruption of the outer layers.

Several uveitic conditions such as placoid syphilitic chorioretinopathy, multiple evanescent white dot syndrome (MEWDS), acute posterior multifocal placoid pigment epitheliopathy (APMPPE), multifocal choroiditis (MFC), birdshot chorioretinopathy (BC), Vogt-Koyanagi-Harada (VKH), serpiginous choroidopathy (SC) and serpiginoid tuberculosis (STB) can target the photoreceptors, the RPE, or the surrounding structures and show unique findings on OCT and OCTA.

In patients with placoid syphilitic chorioretinopathy, disruption of the inner segment/outer segment band (ellipsoid zone according to the currently accepted nomenclature $)^{52}$ and nodular thickening of the RPE with loss of the linear outer segment/RPE junction (interdigitation zone according to the currently accepted nomenclature) ${ }^{52}$ are common findings on OCT scans (Figure 9A). ${ }^{53}$ Subretinal fluid is also frequently detected. In most cases, these alterations disappear with treatment and good vision recovery. A permanent loss of function is seen in cases of persistent photoreceptors damage. $^{53}$

In acute MEWDS lesions, focal disruption of the junction between the RPE and the photoreceptor outer segments, along with hyper-reflectivity of the outer nuclear layer, occurs (Figure 9B). These changes are not associated with any OCTA alterations at the level of the choriocapillaris and disappear with no need for treatment within weeks. ${ }^{54}$

In patients with $\mathrm{BC}$, acute choroidal lesions are composed of infiltrates of epithelioid cells, and therefore they do not show alterations of the choriocapillaris flow on OCTA. ${ }^{5}$ However, if not promptly treated, these lesions evolve to atrophy of the stroma and the overlying choriocapillaris, inducing blood flow impairment that is detectable on OCTA. ${ }^{55}$ Choriocapillaris hypo-perfusion is often accompanied by disruption and atrophy of the overlying RPE and outer retinal layers. ${ }^{56}$

Eyes with MFC typically have round-shaped hyperreflective lesions that break through the RPE and extend into the outer retinal layers. The interruption of the RPE allows more OCT signal to penetrate the choroid, which therefore appears hyper-reflective underneath the lesion (Figure 9C). ${ }^{57}$ OCTA can help in distinguishing these inflammatory foci from iCNVs that commonly complicate MFC. In fact, MFC lesions do not show any internal flow on OCTA, in contrast to iCNV where blood flow is clearly visible within the neovascular network. ${ }^{58}$

Different mechanisms, including RPE dysfunction, choroidal outflow impairment, breakdown of the outer BRB, and leakage from iCNVs can lead to the accumulation of 

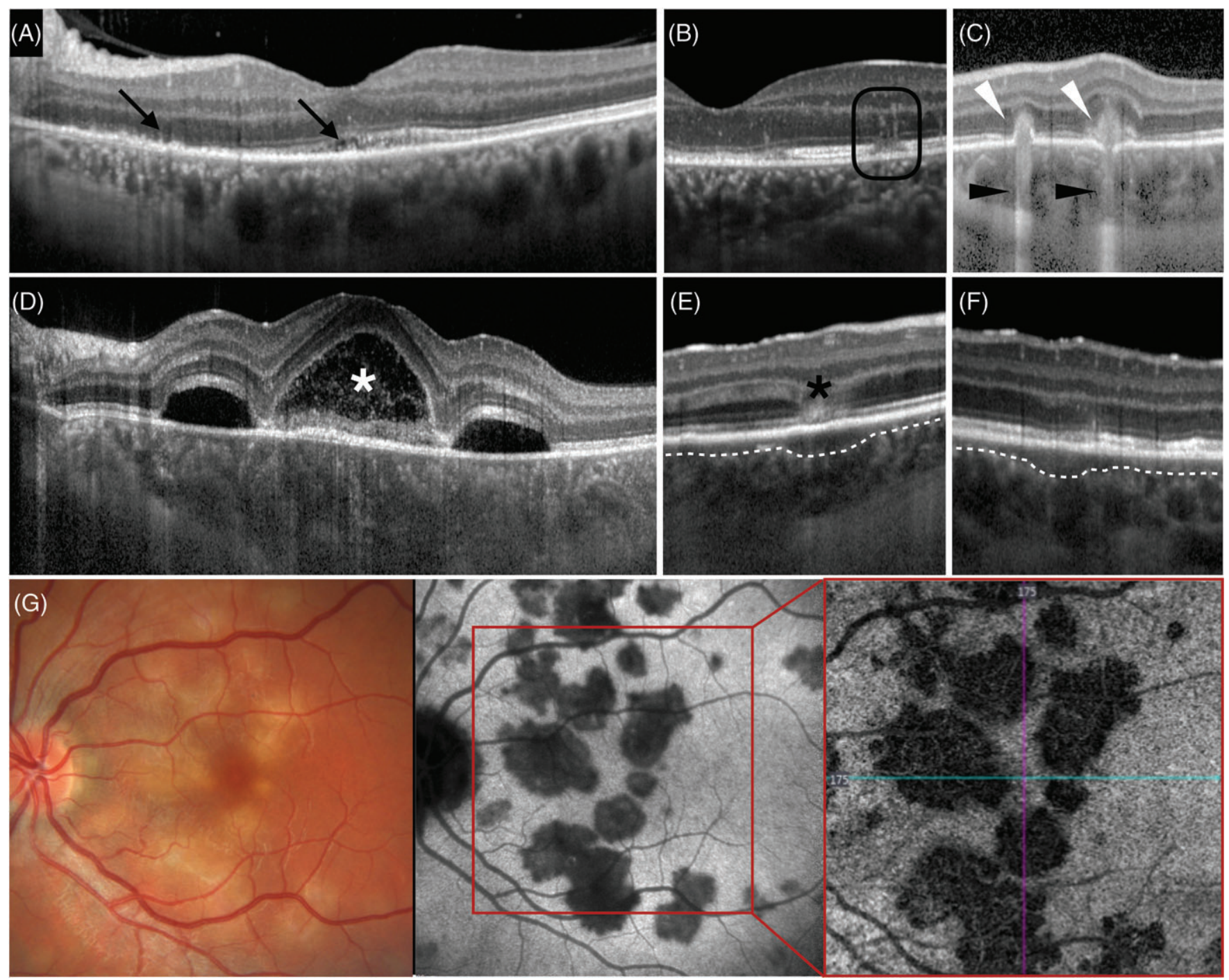

FIGURE 9 Outer retina and choriocapillaris findings on optical coherence tomography (OCT) and OCT angiography (OCTA) in patients with uveitis. (A) Diffuse disruption of the outer retinal layers in a patient with placoid syphilitic chorioretinopathy. (B) Focal disruption and increased reflectivity of the outer retinal layers in multiple evanescent white dot syndrome. (C) Typical outer retinal hyper-reflective lesions breaking through the retinal pigment epithelium (white arrowheads), inducing an increased transmission effect (black arrowheads) in a patient with multifocal choroiditis. (D) Multiple pockets of subretinal fluid in a patient with active Vogt-Koyanagi-Harada disease. Note the typical hyper-reflective content of the fluid (white asterisk). (E, F) Focal thickening of the choriocapillaris (separated from the rest of the choroid with a white dashed line) with (E) and without (F) increased outer retinal reflectivity in a patient with acute posterior multifocal placoid pigment epitheliopathy (APMPPE). The lesion in E (black asterisk) will likely undergo atrophy while that in F will probably disappear without scars. (G) Fundus photography, indocyanine green angiography (ICGA), and OCTA in a patient with acute APMPPE. The hypo-perfusion of the choriocapillaris typical of this condition is clearly visible on OCTA with an almost perfect overlap with that detected by ICGA

fluid between the RPE and the retina. ${ }^{4}$ Subretinal fluid can be easily visualized and monitored with structural OCT, but usually it is not suggestive of a specific aetiology. However, the presence of hyper-reflective septa and the consequent formation of pockets of fluid within the subretinal space is strongly suggestive for acute VKH disease (Figure 9D). ${ }^{59}$

APMPPE and SC are characterized by choriocapillaris hypo-perfusion, demonstrated by indocyanine green angiography (ICGA). These alterations are visible on structural OCT as hypo-reflective focal thickenings and loss of the physiological hyper-reflective dots at the level of the choriocapillaris (Figure 9E,F). Overlying retinal layers may appear normal (Figure 9F) with a consequent good prognosis. Alternatively, the layers demonstrate hyper-reflective changes and disruption (Figure 9E) and undergo atrophy despite treatment. ${ }^{60}$ Similar findings have been reported in STB and VKH, where OCTA demonstrated discrete areas of diminished or absent flow suggestive of choriocapillaris hypoperfusion (Figure 9G). ${ }^{61,62}$

\section{1 | OCT/OCTA OF THE CHOROID IN UVEITIS}

Structural OCT shows that inflammation within the eye is characterized by choroidal thickening, which is due to increased blood flow during the acute stage of the inflammatory process. Variations in choroidal thickness can be 
consequently used to monitor the response to treatment or recurrences. In addition, some features of the choroid visible on OCT scans can suggest specific aetiologies.

The presence of a hypo-reflective round-shaped area with homogeneous content in the choroidal stroma and which generates a cone of increased transmission of the OCT signal through the underlying structures suggests the presence of a choroidal granuloma (Figure 10A). ${ }^{63}$ These lesions are typical of certain types of granulomatous uveitis such as intraocular tuberculosis, sarcoidosis and VKH and can be followed over time on EDI-OCT or SS-OCT scans. in fact OCT was reported to be more sensitive than ICGA in detecting early changes of these lesions in response to treatment. ${ }^{64}$ Large choroidal granulomas can compress the surrounding vasculature, impairing the choroidal blood flow and appearing as areas of flow void on OCTA. ${ }^{5}$

VKH disease is characterized by an inflammatory reaction against melanocytes, and the choroid is the primary site of inflammation. The structural manifestation appears as a massive choroidal thickening as typically seen on EDI-OCT (Figure 10B). ${ }^{65}$ In contrast, a focal thickening of the choroid is present in toxoplasmic retinochoroiditis (Figure 10C). In this entity, the choroidal thickness increases mainly in the areas where the parasites are directly attacking the choroidal and the retinal tissue. The most common finding is focal choroidal thickening with disruption of the physiological choroidal architecture in association with an overlying area of full thickness retinitis. ${ }^{38}$ Finally, a selective enlargement of the Sattler's layer may indicate a diagnosis of sarcoid-related uveitis. ${ }^{66}$ This differentiation may be helpful in distinguishing patients with this disease from other types of granulomatous uveitis.

\section{I OCT/OCTA OF INFLAMMATORY CHOROIDAL NEOVASCULARIZATION}

Uveitis can be complicated by the development of iCNVs, mostly affecting the macular region. The presence of a choroidal neovascularization, even when promptly treated, can lead to severe irreversible vision loss. ${ }^{67}$ The uveitic forms more commonly associated with this complication are punctate inner choroidopathy, ${ }^{68,69}$ MFC, APMPPE, BC, MEWDS and acute zonal occult outer retinopathy. ${ }^{67}$ However, virtually any inflammatory ocular disease can develop an iCNV.

Structural OCT is used as a routine imaging modality to diagnose iCNVs and to assess the activity of the neovascularization. ${ }^{70}$ In addition, OCTA findings can provide critical diagnostic differentiation between iCNVs and other inflammatory non-neovascular lesions. Historically, the identification of iCNVs and inflammatory non-neovascular lesions was based on the use of invasive dye-based procedures such as fundus fluorescein angiography. However, dye leakage can occur from both iCVNs and non-neovascular inflammatory foci (Figure 11A-C). OCTA can clearly demonstrate the presence of the neovascular network forming the iCNV (Figure 11D), a feature that is not present in other types of lesions. ${ }^{71}$

The majority of iCNVs are located above the RPE in the subretinal space, similar to the so-called type 2 or classic lesions of exudative age-related macular degeneration. ${ }^{70}$ On structural OCT scans, an iCNV appears as an accumulation of hyper-reflective material seated between the disrupted RPE and the outer retinal layers, rarely accompanied by a sub-RPE component (Figure 12A). ${ }^{72}$ In addition, active
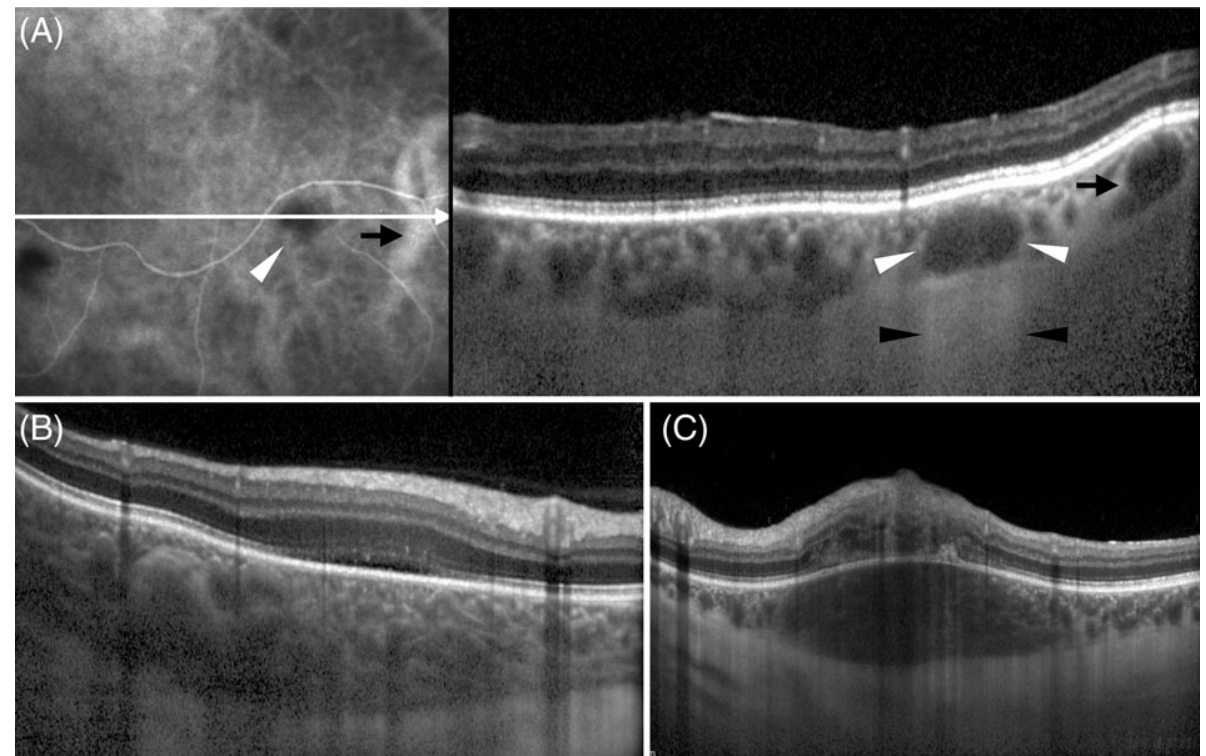

FIGURE 10 Choroidal findings on optical coherence tomography (OCT) in patients with uveitis. While choroidal thickening occurs quite often in patients with uveitis, different patterns can help in the differential diagnosis. (A) Choroidal granulomas, visible on indocyanine green angiography as hypo-reflective lesions, are seen on OCT as round-shaped hypo-reflective formations within the choroid (white arrowhead), resembling large choroidal vessels (black arrow). The presence of an increased transmission effect underneath the granulomas (black arrowheads) distinguishes the lesions from normal choroidal vessels. (B) A massive diffuse thickening of the choroid is common in acute Vogt-Koyanagi-Harada disease, while focal choroidal thickening (C) is usually suggestive of toxoplasmic chorioretinitis 


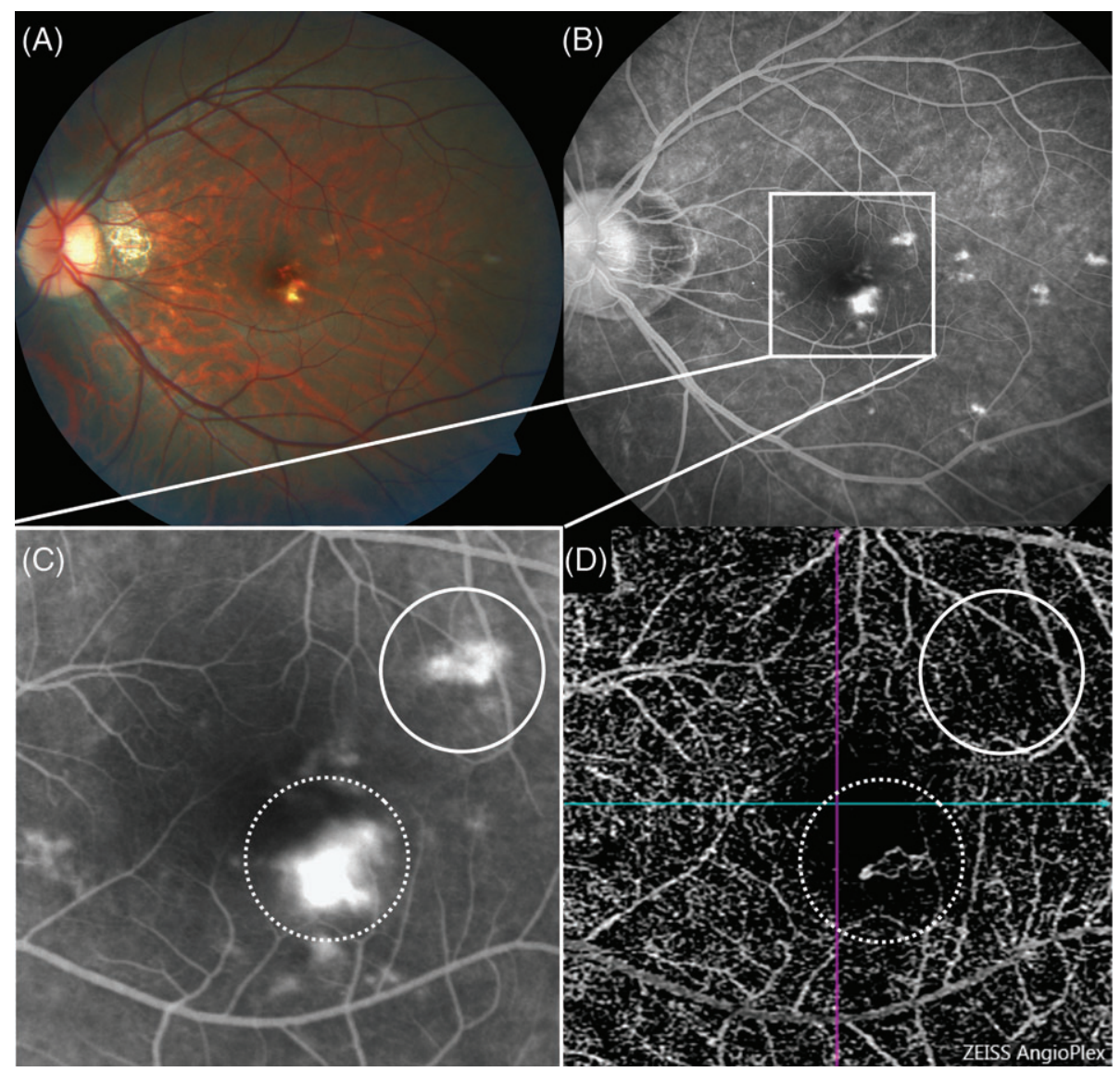

FIGURE 11 Multimodal imaging in a patient affected by acute punctate inner choroidopathy (PIC) complicated by inflammatory choroidal neovascularization (iCNV). (A) On fundus photography, multiple small yellowish lesions are present at the posterior pole. (B) The lesions are better seen on fundus fluorescein angiography (FFA) where they show dye leakage. (C) Due to the presence of leakage, it is hard to differentiate PIC inflammatory foci from iCNV that can complicate the clinical picture. (D) OCTA enables differentiation of the iCNV, which is characterized by the presence of a neovascular network (white dotted circle), from the inflammatory focus, which is characterized by leakage on FFA without neovessels (white circle)

lesions exhibit signs of exudation such as subretinal fluid, subretinal hyper-reflective material, retinal thickening, intraretinal cysts, and shadowing on the underlying structures. In contrast, inactive iCNVs appear as hyper-reflective lesions with clearly marked edges and the absence of sub- or intraretinal fluid. $^{70}$

A peculiar OCT feature that can help in the differential diagnosis between iCNVs and CNVs related to other causes is the so called "pitchfork sign" described in 2013 by Hoang et al. ${ }^{73}$ This consists of multiple, hyper-reflective, vertical projections that extend from the $\mathrm{iCNV}$ area into the outer retinal space.

In most OCTA scans, iCNV appears as a wellcircumscribed neovascular network, typically located at the level of the avascular zone (outer retina RPE). ${ }^{58,69,71}$ Due to alterations in the normal anatomy of the retinal layers induced by the exudative process, the use of automated segmentation software can fail. ${ }^{74}$ Manual adjustments of the reference segmentation line and plane are often necessary to accurately define the neovascular network and differentiate it from superficial retinal vessels and underlying choroidal structures. A good approach while reviewing OCTA scans is to evaluate the flow both on the B-scan and the coronal plane reconstruction (Figure 12B-F). As the B-scans do not need any segmentation to be interpreted, the evaluation of the flow on these images may facilitate the lesion detection and avoid misdiagnosis due to projection artefacts. ${ }^{5}$

While multimodal imaging remains the gold standard approach to comprehensively investigate uveitic patients, combined OCT and OCTA are essential techniques for the detection and management of iCNVs. OCT allows a careful evaluation of the CNV activity and OCTA is extremely helpful in differentiating iCNVs from other non-neovascular inflammatory lesions. ${ }^{70}$

\section{3 | FUTURE DIRECTIONS: OCT/OCTA FOR OBJECTIVE GRADING OF INTRAOCULAR INFLAMMATION}

One of the greatest unmet needs in uveitis management is the objective evaluation of the intraocular inflammation. Clinical grading scales developed by the Standardized Uveitis Nomenclature group decades ago are still the gold standard techniques to assess and grade anterior chamber cells, flare and vitreous haze. ${ }^{20}$ These reference scales are of great 

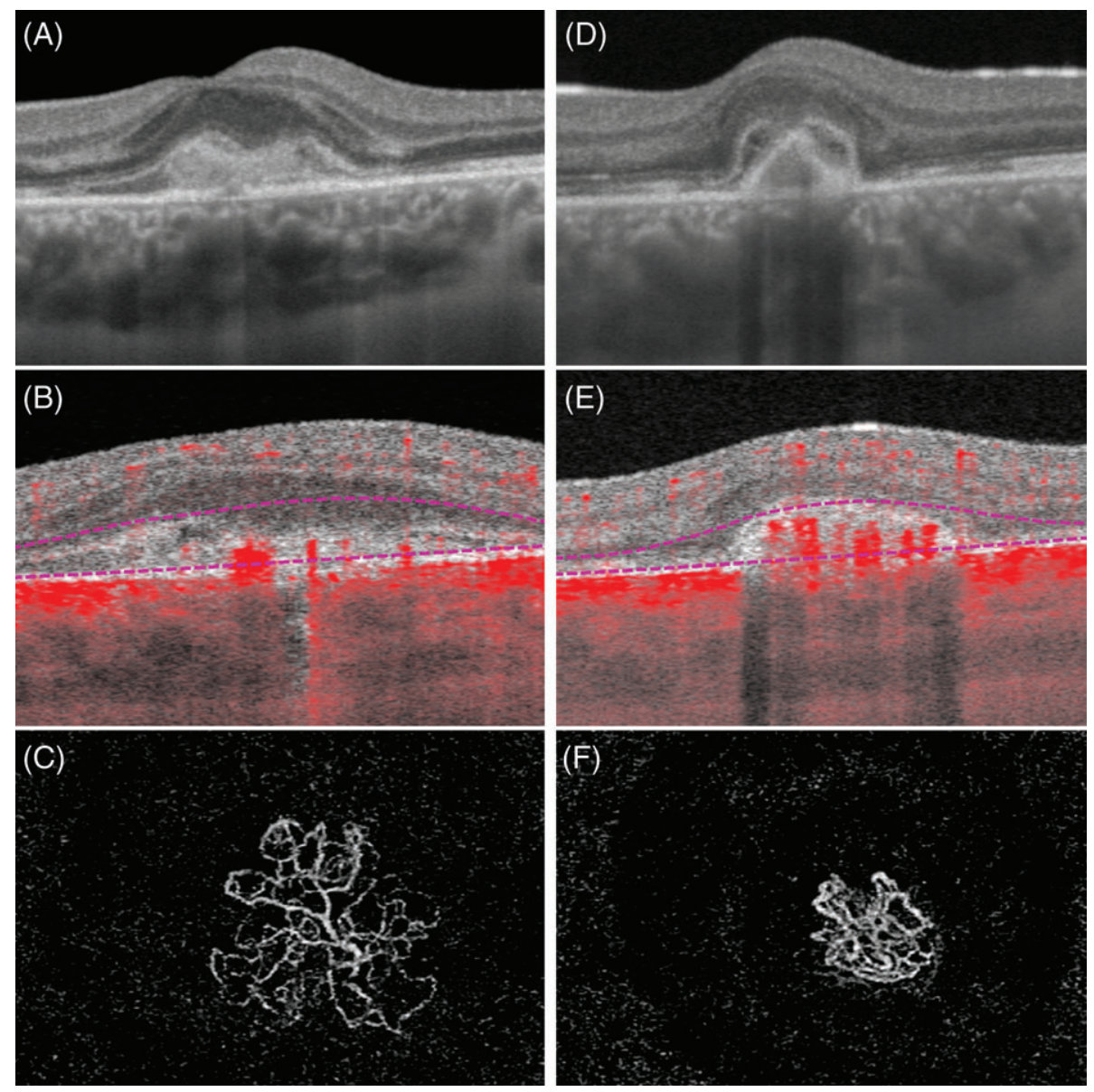

FIGURE 12 Optical coherence tomography (OCT) and OCT angiography (OCTA) findings in inflammatory choroidal neovascularization (iCNV). (A) The active $\mathrm{iCNV}$ appears as a collection of hyper-reflective material spreading between the retinal pigment epithelium (RPE) and the neuroretina on the structural OCT scan. (B) The corresponding OCTA B-scan shows the presence of pathological flow coded in red above the RPE. (C) The OCTA coronal plane visualizes the entire neovascular network with multiple branches. (D) The same lesion appears smaller and better defined on structural OCT 1 month after treatment with anti-vascular endothelial growth factors. (E) The flow signal within the lesion is still visible on the corresponding OCTA B-scan. (F) However, the OCTA reconstruction along the coronal plane allows appreciation of the contraction of the neovascular branches and an overall reduction in the lesion size

value and have been used for the management of uveitic eyes for the last 20 years. However, they suffer from some major limitations, including low inter-operator agreement, poor repeatability, and the lack of a continuous scale. ${ }^{75}$

Recently, new techniques based on the analysis of OCT images have been proposed to overcome the limitations of the clinical scales and to accurately grade intraocular inflammation. Sections of the anterior chamber obtained with ASOCT have been used to count the number of cells and more recently evaluate aqueous flare. ${ }^{11}$ Vitreous haze has been reliably measured on posterior segment OCT scans. ${ }^{12}$ In addition, new parameters such as the choroidal vascularity index (the ratio between vessel lumina and the total choroidal area $)^{76}$ or the vessel density measured by OCTA $^{5}$ have been proposed as adjunctive tools to objectively evaluate the inflammatory status of uveitic eyes.

The near future will likely see a paradigm shift in the grading of intraocular inflammation with objective measurements being performed in a fully, or at least partially, automated way on digital images such as OCT scans. This will allow better management of patients in the everyday clinical practice and a higher accuracy in the evaluation of new drug efficacy in clinical trials. ${ }^{75}$

\section{1 | Conclusions}

OCT has dramatically changed the management of several ocular diseases, including uveitis. Currently, most of the common complications of intraocular inflammation such as macular oedema, epiretinal membranes and iCNVs are routinely diagnosed and followed by OCT/OCTA scans. However, uveitis specialists can obtain much more useful information from tomographic scans, as highlighted in this review. A simple OCT scan through an inflammatory lesion can tell the depth of the alteration, which ocular structures are involved, and with what pattern of changes, providing help in the differential diagnosis, the prediction of clinical outcomes and the patient's follow up. With the development of new automated techniques to objectively grade intraocular inflammation on OCT scans, the use of this imaging 
technique will further increase over time and become even more essential in the management of uveitis.

\section{CONFLICTS OF INTEREST}

None declared.

\section{ORCID}

\section{Alessandro Invernizzi (D) https://orcid.org/0000-0003-3400- 1987}

\section{REFERENCES}

1. Drexler W, Fujimoto JG. State-of-the-art retinal optical coherence tomography. Prog Retin Eye Res. 2008;27:45-88.

2. Spaide RF, Fujimoto JG, Waheed NK, Sadda SR, Staurenghi G. Optical coherence tomography angiography. Prog Retin Eye Res. 2018;64:1-55.

3. Stephen C, Foster MD, Albert T, Vitale MD. Diagnosis and Treatment of Uveitis. Vol 1. New Delhi: Jaypee Brothers; 2013.

4. Onal S, Tugal-Tutkun I, Neri P, Herbort CP. Optical coherence tomography imaging in uveitis. Int Ophthalmol. 2014;34:401-435.

5. Pichi F, Sarraf D, Arepalli S, et al. The application of optical coherence tomography angiography in uveitis and inflammatory eye diseases. Prog Retin Eye Res. 2017;59:178-201.

6. Margolis R, Spaide RF. A pilot study of enhanced depth imaging optical coherence tomography of the choroid in normal eyes. Am J Ophthalmol. 2009; 147:811-815.

7. Mrejen S, Spaide RF. Optical coherence tomography: imaging of the choroid and beyond. Surv Ophthalmol. 2013;58:387-429.

8. Puliafito CA, Hee MR, Lin CP, et al. Imaging of macular diseases with optical coherence tomography. Ophthalmology. 1995;102:217-229.

9. Invernizzi A, Giani A, Cigada M, Staurenghi G. Retrobulbar structure visualization with enhanced depth imaging optical coherence tomography. Invest Ophthalmol Vis Sci. 2013;54:2678-2684.

10. Spaide RF, Fujimoto JG, Waheed NK. Image artifacts in optical coherence tomography angiography. Retina. 2015;35:2163-2180.

11. Invernizzi A, Marchi S, Aldigeri R, et al. Objective quantification of anterior chamber inflammation: measuring cells and flare by anterior segment optical coherence tomography. Ophthalmology. 2017;124:1670-1677.

12. Keane PA, Karampelas M, Sim DA, et al. Objective measurement of vitreous inflammation using optical coherence tomography. Ophthalmology. 2014;121:1706-1714.

13. Kim AY, Rodger DC, Shahidzadeh A, et al. Quantifying retinal microvascular changes in uveitis using spectral-domain optical coherence tomography angiography. Am J Ophthalmol. 2016;171:101-112.

14. Gabriel M, Kruger R, Shams-Mafi F, et al. Mapping retinal and choroidal thickness in unilateral nongranulomatous acute anterior uveitis using threedimensional 1060-nm optical coherence tomography. Invest Ophthalmol Vis Sci. 2017;58:4778-4783.

15. Moore DB, Jaffe GJ, Asrani S. Retinal nerve fiber layer thickness measurements: uveitis, a major confounding factor. Ophthalmology. 2015;122: 511-517.

16. Rougier MB, Le Goff M, Korobelnik JF. Optical coherence tomography angiography at the acute phase of optic disc edema. Eye Vision. 2018;5:15.

17. de Smet MD, Okada AA. Cystoid macular edema in uveitis. Dev Ophthalmol. 2010;47:136-147.

18. Antcliff RJ, Stanford MR, Chauhan DS, et al. Comparison between optical coherence tomography and fundus fluorescein angiography for the detection of cystoid macular edema in patients with uveitis. Ophthalmology. 2000; 107:593-599.

19. Hunter RS, Skondra D, Papaliodis G, Sobrin L. Role of OCT in the diagnosis and management of macular edema from uveitis. Semin Ophthalmol. 2012;27:236-241.

20. Jabs DA, Nussenblatt RB, Rosenbaum JT. Standardization of uveitis nomenclature for reporting clinical data. Results of the First International Workshop. Am J Ophthalmol. 2005;140:509-516.
21. Agarwal A, Ashokkumar D, Jacob S, Agarwal A, Saravanan Y. High-speed optical coherence tomography for imaging anterior chamber inflammatory reaction in uveitis: clinical correlation and grading. Am J Ophthalmol. 2009; 147:413-6.e3

22. Kobayashi R, Hashida N, Soma T, et al. Clinical findings of anterior segment spectral domain optical coherence tomography images in cytomegalovirus corneal endotheliitis. Cornea. 2017;36:411-414.

23. Mahendradas $P$, Avadhani $K$, Ramachandran $S$, Srinivas $S$, Naik $M$, Shetty KB. Anterior segment optical coherence tomography findings of iris granulomas in Hansen's disease: a case report. J Ophthalmic Inflamm Infect. 2013;3:36.

24. Basarir B, Altan C, Pinarci EY, Celik U, Satana B, Demirok A. Analysis of iris structure and iridocorneal angle parameters with anterior segment optical coherence tomography in Fuchs' uveitis syndrome. Int Ophthalmol. 2013; 33:245-250

25. Invernizzi A, Cigada M, Savoldi L, Cavuto S, Fontana L, Cimino L. In vivo analysis of the iris thickness by spectral domain optical coherence tomography. Br J Ophthalmol. 2014;98:1245-1249.

26. Lai I, Mak H, Lai G, Yu M, Lam DS, Leung CK. Anterior chamber angle imaging with swept-source optical coherence tomography: measuring peripheral anterior synechia in glaucoma. Ophthalmology. 2013;120:11441149.

27. Kim M, Choi SY, Park YH. Analysis of choroidal and central foveal thicknesses in acute anterior uveitis by enhanced-depth imaging optical coherence tomography. BMC Ophthalmol. 2017;17:225.

28. Shoughy SS, Jaroudi MO, Kozak I, Tabbara KF. Optical coherence tomography in the diagnosis of scleritis and episcleritis. Am J Ophthalmol. 2015; 159:1045-9.e1.

29. Axmann S, Ebneter A, Zinkernagel MS. Imaging of the sclera in patients with scleritis and episcleritis using anterior segment optical coherence tomography. Ocul Immunol Inflamm. 2016;24:29-34.

30. Kuroda Y, Uji A, Morooka S, Nishijima K, Yoshimura N. Morphological features in anterior scleral inflammation using swept-source optical coherence tomography with multiple B-scan averaging. Br J Ophthalmol. 2017; 101:411-417.

31. Akagi T, Uji A, Huang AS, et al. Conjunctival and intrascleral vasculatures assessed using anterior-segment optical coherence tomography angiography in normal eyes. Am J Ophthalmol. 2018;196:1-9.

32. Pang CE, Freund KB, Engelbert M. Enhanced vitreous imaging technique with spectral-domain optical coherence tomography for evaluation of posterior vitreous detachment. JAMA Ophthalmol. 2014;132:1148-1150.

33. Spaide RF. Visualization of the posterior vitreous with dynamic focusing and windowed averaging swept source optical coherence tomography. Am J Ophthalmol. 2014;158:1267-1274.

34. Saito M, Barbazetto IA, Spaide RF. Intravitreal cellular infiltrate imaged as punctate spots by spectral-domain optical coherence tomography in eyes with posterior segment inflammatory disease. Retina. 2013;33:559-565.

35. Mahendradas P, Madhu S, Kawali A, et al. Enhanced vitreous imaging in uveitis. Ocul Immunol Inflamm. 2017;1-7. https://doi.org/10.1080/09273948. 2017.1360501

36. Invernizzi A, Agarwal A, Ravera V, Oldani M, Staurenghi G, Viola F. Optical coherence tomography findings in cytomegalovirus retinitis: a longitudinal study. Retina. 2017; 38:108-117. https://doi.org/10.1097/IAE.0000000000 001503 .

37. Goldenberg D, Goldstein M, Loewenstein A, Habot-Wilner Z. Vitreal, retinal, and choroidal findings in active and scarred toxoplasmosis lesions: a prospective study by spectral-domain optical coherence tomography. Graefes Arch Clin Exp Ophthalmol. 2013;251:2037-2045.

38. Invernizzi A, Agarwal AK, Ravera V, et al. Comparing optical coherence tomography findings in different aetiologies of infectious necrotising retinitis. Br J Ophthalmol. 2018;102:433-437.

39. Invernizzi A, Symes R, Miserocchi E, et al. Spectral domain optical coherence tomography findings in endogenous candida endophthalmitis and their clinical relevance. Retina. 2017;38:1011-1018. https://doi.org/10.1097/IAE. 0000000000001630.

40. Invernizzi A, Cozzi M, Symes R, Pellegrini M, Staurenghi G. Ocular neovascularization in endogenous candida endophthalmitis: using multimodal imaging to understand different pathogenic pathways. Retina. 2018;38: e17-e19.

41. Wintergerst MWM, Pfau M, Muller PL, et al. Optical coherence tomography angiography in intermediate uveitis. Am J Ophthalmol. 2018;194:35-45. 
42. Walton RC, Ashmore ED. Retinal vasculitis. Curr Opin Ophthalmol. 2003; 14:413-419.

43. Thomas AS, Hatef AL, Stinnett SS, Keenan RT, Jaffe GJ. Perivascular thickening on optical coherence tomography as a marker of inflammation in birdshot retinochoroiditis. Retina. 2018;1. https://doi.org/10.1097/IAE. 0000000000002038

44. Knickelbein JE, Tucker W, Kodati S, Akanda M, Sen HN. Non-invasive method of monitoring retinal vasculitis in patients with birdshot chorioretinopathy using optical coherence tomography. Br J Ophthalmol. 2018;102: 815-820.

45. Gomez ML, Mojana F, Bartsch DU, Freeman WR. Imaging of long-term retinal damage after resolved cotton wool spots. Ophthalmology. 2009;116: 2407-2414.

46. Falkenberry SM, Ip MS, Blodi BA, Gunther JB. Optical coherence tomography findings in central retinal artery occlusion. Ophthalmic Surg Lasers Imaging. 2006;37:502-505.

47. Kurup SP, Khan S, Gill MK. Spectral domain optical coherence tomography in the evaluation and management of infectious retinitis. Retina. 2014;34: 2233-2241.

48. Tugal-Tutkun I, Ozdal PC, Oray M, Onal S. Review for diagnostics of the year: multimodal imaging in Behcet uveitis. Ocul Immunol Inflamm. 2017; 25:7-19.

49. Khairallah M, Kahloun R, Gargouri S, et al. Swept-Source Optical Coherence Tomography Angiography in West Nile Virus Chorioretinitis and Associated Occlusive Retinal Vasculitis. Ophthalmic Surg Lasers Imaging Retina. 2017;48:672-675

50. Khairallah M, Abroug N, Khochtali S, et al. Optical coherence tomography angiography in patients with Behcet uveitis. Retina. 2017;37:1678-1691.

51. Pichi F, Srivastava SK, Levinson A, Baynes KM, Traut C, Lowder CY. A focal chorioretinal bartonella lesion analyzed by optical coherence tomography angiography. Ophthalmic Surg Lasers Imaging Retina. 2016;47:585-588.

52. Staurenghi G, Sadda S, Chakravarthy U, Spaide RF. Proposed lexicon for anatomic landmarks in normal posterior segment spectral-domain optical coherence tomography: the IN-OCT consensus. Ophthalmology. 2014;121: 1572-1578.

53. Pichi F, Ciardella AP, Cunningham ET Jr, et al. Spectral domain optical coherence tomography findings in patients with acute syphilitic posterior placoid chorioretinopathy. Retina. 2014;34:373-384.

54. Pichi F, Srvivastava SK, Chexal S, et al. En face optical coherence tomography and optical coherence tomography angiography of multiple evanescent white dot syndrome: new insights into pathogenesis. Retina. 2016;36(suppl 1):S178-S188.

55. de Carlo TE, Bonini Filho MA, Adhi M, Duker JS. Retinal and choroidal vasculature in birdshot chorioretinopathy analyzed using spectral domain optical coherence tomography angiography. Retina. 2015;35:2392-2399.

56. Witkin AJ, Duker JS, Ko TH, Fujimoto JG, Schuman JS. Ultrahigh resolution optical coherence tomography of birdshot retinochoroidopathy. $\mathrm{Br} \mathrm{J}$ Ophthalmol. 2005;89:1660-1661.

57. Vance SK, Khan S, Klancnik JM, Freund KB. Characteristic spectraldomain optical coherence tomography findings of multifocal choroiditis. Retina. 2011;31:717-723.

58. Cheng L, Chen X, Weng S, et al. Spectral-domain optical coherence tomography angiography findings in multifocal choroiditis with active lesions. Am J Ophthalmol. 2016;169:145-161.

59. Ishihara K, Hangai M, Kita M, Yoshimura N. Acute Vogt-Koyanagi-Harada disease in enhanced spectral-domain optical coherence tomography. Ophthalmology. 2009;116:1799-1807.

60. Invernizzi A, Agarwal A, Cozzi M, Viola F, Nguyen QD, Staurenghi G. Enhanced depth imaging optical coherence tomography features in areas of choriocapillaris hypoperfusion. Retina. 2016;36:2013-2021.
61. Mandadi SKR, Agarwal A, Aggarwal K, et al. Novel findings on optical coherence tomography angiography in patients with tubercular serpiginouslike choroiditis. Retina. 2017;37:1647-1659.

62. Aggarwal K, Agarwal A, Mahajan S, et al. The role of optical coherence tomography angiography in the diagnosis and management of acute VogtKoyanagi-Harada disease. Ocul Immunol Inflamm. 2018;26:142-153.

63. Invernizzi A, Mapelli C, Viola F, et al. Choroidal granulomas visualized by enhanced depth imaging optical coherence tomography. Retina. 2015;35: 525-531.

64. Invernizzi A, Agarwal A, Mapelli C, Nguyen QD, Staurenghi G, Viola F. Longitudinal follow-up of choroidal granulomas using enhanced depth imaging optical coherence tomography. Retina. 2017;37:144-153.

65. Nakayama M, Keino H, Okada AA, et al. Enhanced depth imaging optical coherence tomography of the choroid in Vogt-Koyanagi-Harada disease. Retina. 2012;32:2061-2069.

66. Mehta H, Sim DA, Keane PA, et al. Structural changes of the choroid in sarcoid- and tuberculosis-related granulomatous uveitis. Eye. 2015;29:10601068.

67. Baxter SL, Pistilli M, Pujari SS, et al. Risk of choroidal neovascularization among the uveitides. Am J Ophthalmol. 2013;156:468-77.e2.

68. Channa R, Ibrahim M, Sepah Y, et al. Characterization of macular lesions in punctate inner choroidopathy with spectral domain optical coherence tomography. J Ophthalmic Inflamm Infect. 2012;2:113-120.

69. Levison AL, Baynes KM, Lowder CY, Kaiser PK, Srivastava SK. Choroidal neovascularisation on optical coherence tomography angiography in punctate inner choroidopathy and multifocal choroiditis. Br J Ophthalmol. 2017; 101:616-622.

70. Agarwal A, Invernizzi A, Singh RB, et al. An update on inflammatory choroidal neovascularization: epidemiology, multimodal imaging, and management. J Ophthalmic Inflamm Infect. 2018;8:13.

71. Astroz P, Miere A, Mrejen S, et al. Optical coherence tomography angiography to distinguish choroidal neovascularization from macular inflammatory lesions in multifocal choroiditis. Retina. 2018;38:299-309.

72. Roy R, Saurabh K, Bansal A, Kumar A, Majumdar AK, Paul SS. Inflammatory choroidal neovascularization in Indian eyes: etiology, clinical features, and outcomes to anti-vascular endothelial growth factor. Indian J Ophthalmol. 2017;65:295-300.

73. Hoang QV, Cunningham ET Jr, Sorenson JA, Freund KB. The "pitchfork sign" a distinctive optical coherence tomography finding in inflammatory choroidal neovascularization. Retina. 2013;33:1049-1055.

74. Pichi F, Sarraf D, Morara M, Mazumdar S, Neri P, Gupta V. Pearls and pitfalls of optical coherence tomography angiography in the multimodal evaluation of uveitis. J Ophthalmic Inflamm Infect. 2017;7:20.

75. Denniston AK, Keane PA, Srivastava SK. Biomarkers and surrogate endpoints in uveitis: the impact of quantitative imaging. Invest Ophthalmol Vis Sci. 2017;58:Bio131-Bio140.

76. Agrawal R, Salman M, Tan KA, et al. Choroidal Vascularity Index (CVI)a novel optical coherence tomography parameter for monitoring patients with panuveitis? PLoS One. 2016;11:e0146344.

How to cite this article: Invernizzi A, Cozzi M, Staurenghi G. Optical coherence tomography and optical coherence tomography angiography in uveitis: A review. Clin. Experiment. Ophthalmol. 2019;1-15. https://doi.org/10.1111/ceo.13470 